\title{
Apuração do Capital Social Acumulado a partir de Interações Sociais em Páginas Institucionais no Facebook - Resumo Estendido - CTDSI/CTCCSI 2021
}

\author{
Kaique Matheus R. Cunha ${ }^{1}$, Orientador: Alan Keller Gomes ${ }^{1}$ \\ ${ }^{1}$ Instituto Federal de Educação, Ciência e Tecnologia de Goiás (IFG) - Câmpus Inhumas \\ Av. Universitária, s/nº, Vale das Goiabeiras, Inhumas-GO - Brasil - CEP: 75400-000 \\ ifgkaique@gmail.com, alan.gomes@ifg.edu.br
}

\section{Visão Geral do Tema}

Neste resumo estendido é apresentada uma síntese do Trabalho de Conclusão de Curso (TCC) intitulado como "Mensuração do Capital Social Acumulado a partir de Interações Sociais em Páginas Institucionais no Facebook", no qual são apresentados procedimentos voltados para a apuração do Capital Social (CS) contido em fanpages institucionais na rede social Facebook. Esse trabalho foi publicado no IX Brazilian Workshop on Social Network Analysis and Mining (BraSNAM 2020) [Cunha e Gomes 2020].

O estudo do Capital Social tem ganhado uma atenção crescente da comunidade científica que desenvolve pesquisas voltadas para a análise de aspectos e impactos tecnológicos e sociais de Sistemas de Informação (SI), principalmente, os sistemas que são mediados pelo uso de tecnologias interativas, tais como as Redes Sociais Online (RSO) [Dhar e Bose 2019]. Dentro de uma visão sociotécnica de SI [Abreu e Abreu 2002], as RSO são compostas de uma parte técnica e outra social que requerem investimentos substanciais de ordem social, organizacional e intelectual para, dentre outros, viabilizar o acúmulo de CS no meio digital [Law e Chang 2008].

Pessoas, marcas e instituições que estão presentes nas RSO estão cada vez mais interessadas em saber de que maneira o Capital Social acumulado nesses SI pode refletir no acúmulo do CS no mundo físico, principalmente, a partir da comunicação e interação direta com a rede de conexões da vida real, que se expandem, se fortalecem e surgem novas no meio digital [Silva et al. 2020]. A mensuração do CS acumulado nas RSO é um passo importante para a adoção de estratégias de manutenção e aumento desse tipo de capital que se reflete no CS acumulado no mundo offline.

Segundo o sociólogo Pierre Bourdieu, o capital acumulado a partir das conexões sociais que os agentes estabelecem em um determinado campo é denominado de Capital Social [Bourdieu 1986]. O Capital Social é acumulado a partir das práticas de sociabilidade em rede, que se cristalizam por meio das trocas realizadas entre os agentes presentes no campo. Nesta pesquisa, as práticas de sociabilidade em rede correspondem à execução de funcionalidades como Publicar, Curtir, Compartilhar, Comentar; e ainda, agentes são Páginas Institucionais (fanpages) e o campo é a rede Facebook.

Apesar de indicadores do acúmulo desse tipo de capital terem sido estudados, principalmente, a partir da estrutura da rede de conexões de cada agente, não há consenso em como o CS pode ser medido. No intuito de contribuir para a superação dessa lacuna, o objetivo geral do presente trabalho é apresentar um conjunto de procedimentos voltados 
para a apuração do CS acumulado em Páginas Institucionais dentro do Facebook, a partir do número de interações sociais capturadas de cada página.

A pesquisa aqui realizada para a elaboração dos procedimentos de mensuração do CS é de caráter interdisciplinar, pois congrega a área de SI com a área de Sociologia Digital [Ignatow e Robinson 2017]. Diante do exposto, o trabalho aqui apresentado está inserido nas temáticas e traz contribuições aos Grandes Desafios da Pesquisa em SI, especificamente em Challenge 4 - Sociotechnical View of Information Systems: Systemic and Socially Aware Perspective for Information Systems.

\section{Recorte da Pesquisa}

Na Sociologia Digital, as práticas de sociabilidade dos agentes (i.e., página institucional) e sua rede de conexões (i.e., seguidores) no campo (i.e., RSO) são cristalizadas a partir da execução de funcionalidades intrínsecas à rede. Na rede Facebook, logo após uma publicação, as ações Curtir, Comentar e Compartilhar ficam disponíveis e podem ser executadas pelos usuários conectados. Para analisar essas práticas, foi selecionada uma instituição pública de ensino profissional com 13 câmpus. Cada câmpus tem autonomia para gerir sua fanpage seguindo diretrizes internas, e ainda, do governo federal ${ }^{1}$. O público conectado nessas páginas são, em sua imensa maioria, estudantes, professores e demais servidores. Cada página é sempre o único provedor de estímulos sociais e o público pode interagir somente executando as ações mencionadas.

\section{Etapas da Pesquisa}

Na pesquisa aqui apresentada, a abordagem metodológica é empírica, quantitativa e descritiva. O método de pesquisa é a experimentação. Os passos empregados são:

1. Identificação dos dados a serem capturados

2. Coleta, transformação e carregamento

3. Extração e mensuração de sequências

4. Validação das mensurações

5. Apuração do Capital Social

Em cada um desses passos foram implementados procedimentos que, no seu conjunto, estão voltados para a apuração do Capital Social de Bourdieu. Maiores detalhes podem ser obtidos em [Cunha e Gomes 2020] na Seção 2.

\section{Síntese dos Resultados}

Os resultados obtidos estão alinhados com as teorias de Bourdieu no que se refere 1) à representação do sistema de trocas entre agentes e sua rede de conexões, 2) à identificação da capacidade (incapacidade) de mobilização da rede de conexões de uma agente, 3) à capacidade (incapacidade) de mobilização de um agente que independe do tamanho da sua rede de conexões, 4) uma grande rede de contatos não garante um grande volume de Capital Social acumulado, ou seja, um volume maior de Capital Social acumulado depende da capacidade (efetiva) de mobilização da rede de conexões do agente, e 5) o Capital Social depende de um esforço incessante de sociabilidade, entretanto, um grande número de interações sociais não provoca necessariamente maior acúmulo de Capital Social, pois depende da efetiva mobilização da rede de conexões. Maiores detalhes podem ser obtidos em [Cunha e Gomes 2020] na Seção 3.

\footnotetext{
${ }^{1}$ Www.governodigital.gov.br/transformacao/compras/orientacoes/orientacoes-redes-sociais-1
} 


\section{Conclusão}

Neste resumo estendido foi apresentada uma síntese do TCC intitulado "Mensuração do Capital Social Acumulado a partir de Interações Sociais em Páginas Institucionais no Facebook", no qual são detalhados um conjunto de procedimentos voltados para a apuração o Capital Social, levando-se em conta o número de interações sociais que ocorrem dentro dessas páginas. O arcabouço teórico de Bourdieu foi utilizado no direcionamento da coleta de dados relativos às práticas de sociabilidade em rede, e ainda, no estabelecimento de um indicador do Capital Social acumulado e do volume de Capital Social acumulado.

O escopo da pesquisa aqui apresentada está limitado a análise das ações executadas dentro de Páginas Institucionais por seguidores dessas páginas. Essas páginas têm a característica peculiar de que o único agente provedor de estímulos para as interações sociais é a página institucional. O campo considerado limita-se a rede social Facebook. Em trabalhos futuros, o conjunto de procedimentos apresentado aqui será usado como base para a proposição nova abordagem de mensuração do Capital Social Bourdieusiano na rede Facebook [Cunha et al. 2021].

\section{Agradecimentos}

Os autores agradecem ao PIBICTI-IFG ${ }^{2}$ e ao $\mathrm{CNPq}^{\text {. }}$

\section{Referências}

Abreu, P. F. e Abreu, A. F. (2002). Sistemas de informações gerenciais: uma abordagem orientada à gestão empresarial. São Paulo: Atlas.

Bourdieu, P. (1986). The forms of capital. In Handbook of Theory and Research for the Sociology of Education, pages 241-258. Greenw. Press, NY,USA.

Cunha, K. M. R., da Costa, R. L., e Gomes, A. K. (2021). A measurement approach to the bourdieusian social capital within facebook institutional pages. In Proceedings of the XVII Brazilian Symposium on Information Systems (SBSI 2021) (accepted). ACM.

Cunha, K. M. R. e Gomes, A. K. (2020). Mensuração do capital social acumulado a partir de interações sociais em páginas institucionais no facebook. In Anais do IX Brazilian Workshop on Social Network Analysis and Mining, BraSNAM 2020, pages 85-96, Cuiabá, MT, Brasil. SBC.

Dhar, S. e Bose, I. (2019). Empirical study of social capital factors formed through digital social networking. In Proceedings of the International Conference on Information Systems, ICIS 2019. AIS eLibrary.

Ignatow, G. e Robinson, L. (2017). Pierre bourdieu: theorizing the digital. Information, Communication \& Society, 20(7):950-966.

Law, S. P.-M. e Chang, M. K. (2008). Fostering knowledge exchange in online communities: a social capital building approach. In Proceedings of the International Conference on Information Systems, ICIS 2008, pages 339-365. AIS eLibrary.

Silva, T. A. d. S., Corrêa, V. S., Vale, G. M. V., e Giglio, E. M. (2020). Influence of social capital offline and online on early-stage entrepreneurs. Revista de Gestão.

\footnotetext{
${ }^{2} \mathrm{http}$ ///www.ifg.edu.br/component/content/article/63-ifg/pro-reitorias/pesquisa-e-pos-graduacao/149iniciacao-cientifica-e-tecnologica
} 